\title{
The general relativistic double polytrope for anisotropic matter.
}

\author{
G. Abellán * and E. Fuenmayor ${ }^{\dagger}$ \\ Departamento de Física, Facultad de Ciencias, \\ Universidad Central de Venezuela, \\ A.P. 47270, Caracas 1041-A, Venezuela. \\ E. Contreras $\ddagger$ \\ Departamento de Física, Colegio de Ciencias e Ingeniería, \\ Universidad San Francisco de Quito, Quito, Ecuador. \\ L. Herrera § \\ Instituto Universitario de Física Fundamental y Matemáticas, \\ Universidad de Salamanca, Salamanca 37007, Spain
}

\begin{abstract}
A general formalism recently proposed to study Newtonian polytropes for anisotropic fluids [1] is here extended to the relativistic regime. Thus, it is assumed that a polytropic equation of state is satisfied by, both, the radial and the trangential pressures of the fluid. Doing so the generalized Lane-Emden equations are obtained and solved. Some specific models are obtained, and their physical properties are discussed.

PACS numbers:
\end{abstract}

\section{INTRODUCTION}

In a recent paper an approach for the study of Newtonian polytropes for anisotropic fluids has been proposed [1. The main idea underlying such an approach consists in complementing the general treatment of Newtonian polytropes for anisotropic matter described in [2] with the additional assumption that both principal stresses satisfy a polytropic equations of state. Doing so we were able to solve the ensuing Lane-Emden equations, for any set of the parameters of the problem.

Polytropic equations of state have a long and a venerable history in astrophysics (see [3, 4] and references therein), and have been extensively used to study the stellar structure under a variety of circumstances.

For a fluid with isotropic pressure, the theory of polytropes is based on the polytropic equation of state, which in the Newtonian case reads

$$
P=K \rho_{0}^{\gamma}=K \rho_{0}^{1+1 / n}
$$

where $P$ and $\rho_{0}$ denote the isotropic pressure and the mass (baryonic) density, respectively. Constants $K, \gamma$, and $n$ are usually called the polytropic constant, polytropic exponent, and polytropic index, respectively.

Once the equation of state (1) is assumed, the whole system is described by an equation (Lane-Emden) that

\footnotetext{
*gabriel.abellan@ciens.ucv.ve

† ernesto.fuenmayor@ciens.ucv.ve

‡econtreras@usfq.edu.ec

$\S$ lherrera@usal.es
}

may be solved for any set of the parameters of the theory.

However we know nowaday that, on the one hand, the pressure isotropy may be a too stringent condition, and on the other hand that pressure anisotropy is produced by many different physical phenomena, of the kind one expects to be present in very compact objects (see [1, 2, 5. and references therein). Besides, as it has been recently proved, the isotropic pressure condition becomes unstable by the presence of physical factors such as dissipation, energy density inhomogeneity and shear [6]. These facts explain the renewed interest in the study of fluids not satisfying the isotropic pressure condition, and justify our interest to extend the theory of polytropes to anisotropic fluids.

Thus, if we assume the fluid pressure to be anisotropic, the two principal stresses (say $P_{r}$ and $P_{\perp}$ ) are unequal and the Newtonian polytrope is characterized by the equation:

$$
P_{r}=K \rho_{0}^{\gamma}=K \rho_{0}^{1+1 / n}
$$

In this case there is an additional degree of freedom and therefore a single equation of state is not enough to integrate the Lane-Emden equation. In order to overcome this underdetermination of the problem, we have assumed in [1], that $P_{\perp}$ also satisfies a polytropic equation of state.

All the above concerns the theory of Newtonian polytropes which applies for self-gravitating objects with a degree of compactness of the order of (or lower than) the corresponding to a white dwarf, for which Newtonian gravity is enough to describe the gravitational interaction. However for more compact objects (e.g. neutron 
stars, super-Chandrasekhar white dwarfs) we have to resort to general relativity.

For all the reasons above, we endeavour in this work to extend the approach developed in [1] to the relativistic regime.

General relativistic polytropes have been extensively studied in the past (see [7] 27] and references therein), in particular a comprehensive framework to describe general relativistic polytropes for anisotropic fluids has been presented in [16].

As it happens in the Newtonian case, the fact that the principal stresses are unequal, produces in the relativistic case too an underdetermination of the problem, requiring to impose an additional condition.

Here we propose to follow the same strategy as in 1], i.e. we shall assume that both the radial $\left(P_{r}\right)$ and tangential $\left(P_{\perp}\right)$ pressures satisfy a polytropic equation of state, i.e.

$$
\begin{aligned}
& P_{r}=K_{r} \rho^{\gamma_{r}}=K_{r} \rho^{1+1 / n_{r}}, \\
& P_{\perp}=K_{\perp} \rho^{\gamma_{\perp}}=K_{\perp} \rho^{1+1 / n_{\perp}},
\end{aligned}
$$

where $\rho$ denotes the energy density. An important remark is in order at this point: when considering the polytropic equation of state within the context of general relativity, two different possibilities arise, leading to the same equation (2) in the Newtonian limit; these are (3) and

$$
P_{r}=K_{r} \rho_{0}^{\gamma_{r}}=K_{r} \rho_{0}^{1+1 / n_{r}} .
$$

Since we are assuming that the tangential pressure $P_{\perp}$ also satisfies a polytropic equation of state, it implies that we have four possible cases leading to the same Newtonian limit. The general treatment is very similar for all these cases and therefore, for simplicity, we shall restrict here to the case described by 3,4 .

The manuscript is organized as follows. In the next section we introduce the main equations and conventions, and briefly review the main aspects of anisotropic polytropes. Next we introduce the polytropic equation of state for both pressures in section III and discuss the numerical results. The last section is devoted to final remarks and conclusions.

\section{THE POLYTROPE FOR ANISOTROPIC FLUID}

\section{A. The field equations and conventions}

We shall consider a static, spherically symmetric distribution of an anisotropic fluid bounded by a surface $\Sigma$. In Schwarzschild-like coordinates, the metric is parametrized as

$$
d s^{2}=e^{\nu} d t^{2}-e^{\lambda} d r^{2}-r^{2}\left(d \theta^{2}+\sin ^{2} d \phi^{2}\right),
$$

where $\nu$ and $\lambda$ are functions of $r$.
The matter content of the sphere is described by the energy-momentum tensor

$$
T_{\mu \nu}=\left(\rho+P_{\perp}\right) u_{\mu} u_{\nu}-P_{\perp} g_{\mu \nu}+\left(P_{r}-P_{\perp}\right) s_{\mu} s_{\nu},
$$

where,

$$
u^{\mu}=\left(e^{-\nu / 2}, 0,0,0\right)
$$

is the four velocity of the fluid, $s^{\mu}$ is defined as

$$
s^{\mu}=\left(0, e^{-\lambda}, 0,0\right),
$$

with the properties $s^{\mu} u_{\mu}=0, s^{\mu} s_{\mu}=-1$. Notice that we are assuming geometric units $c=G=1$.

The metric (6), has to satisfy the Einstein field equations, which are given by

$$
\begin{gathered}
\rho=-\frac{1}{8 \pi}\left[-\frac{1}{r^{2}}+e^{-\lambda}\left(\frac{1}{r^{2}}-\frac{\lambda^{\prime}}{r}\right)\right], \\
P_{r}=-\frac{1}{8 \pi}\left[\frac{1}{r^{2}}-e^{-\lambda}\left(\frac{1}{r^{2}}+\frac{\nu^{\prime}}{r}\right)\right], \\
P_{\perp}=\frac{1}{8 \pi}\left[\frac{e^{-\lambda}}{4}\left(2 \nu^{\prime \prime}+\nu^{\prime 2}-\lambda^{\prime} \nu^{\prime}+2 \frac{\nu^{\prime}-\lambda^{\prime}}{r}\right)\right],
\end{gathered}
$$

where primes denote derivative with respect to $r$.

Outside of the fluid distribution the spacetime is given by the Schwarzschild exterior solution, namely

$$
\begin{aligned}
d s^{2}= & \left(1-\frac{2 M}{r}\right) d t^{2}-\left(1-\frac{2 M}{r}\right)^{-1} d r^{2} \\
& -r^{2}\left(d \theta^{2}+\sin ^{2} d \phi^{2}\right)
\end{aligned}
$$

Furthermore, we require the continuity of the first and the second fundamental form across the boundary surface $r=r_{\Sigma}=$ constant, which implies,

$$
\begin{aligned}
e^{\nu_{\Sigma}} & =1-\frac{2 M}{r_{\Sigma}} \\
e^{-\lambda_{\Sigma}} & =1-\frac{2 M}{r_{\Sigma}} \\
P_{r_{\Sigma}} & =0
\end{aligned}
$$

where the subscript $\Sigma$ indicates that the quantity is evaluated at the boundary surface $\Sigma$.

From the radial component of the conservation law,

$$
\nabla_{\mu} T^{\mu \nu}=0
$$

one obtains the generalized Tolman-OppenheimerVolkoff equation for anisotropic matter which reads,

$$
P_{r}^{\prime}=-\frac{\nu^{\prime}}{2}\left(\rho+P_{r}\right)+\frac{2}{r}\left(P_{\perp}-P_{r}\right) .
$$

Alternatively, using

$$
\nu^{\prime}=2 \frac{m+4 \pi P_{r} r^{3}}{r(r-2 m)},
$$


where the mass function $m$ is as usually defined as

$$
e^{-\lambda}=1-2 m / r
$$

we may rewrite Eq. 18 in the form

$$
P_{r}^{\prime}=-\frac{m+4 \pi r^{3} P_{r}}{r(r-2 m)}\left(\rho+P_{r}\right)+\frac{2}{r} \Delta
$$

where

$$
\Delta=P_{\perp}-P_{r}
$$

measures the anisotropy of the system.

For the physical variables appearing in (21) the following boundary conditions apply

$$
m(0)=0, \quad m(\Sigma)=M, \quad P_{r}\left(r_{\Sigma}\right)=0 .
$$

As already mentioned in the previous section, in order to integrate (21), we shall need an additional condition, besides (3). In this work such condition is (4).

\section{B. Relativistic polytrope for anisotropic fluids}

We shall now expose the basics of the theory of relativistic polytropes for anisotropic matter (for details see [16]).

The starting assumption is to adopt the polytropic equation of state (3) for the radial pressure, i.e.

$$
P_{r}=K \rho^{\gamma_{r}}=K \rho^{1+\frac{1}{n_{r}}} .
$$

As is well known from the general theory of polytropes, there is a bifurcation at the value $\gamma=1$. Thus, the cases $\gamma=1$ and $\gamma \neq 1$ have to be considered separately.

Let us first consider the case $\gamma \neq 1$. Thus, defining the variable $w$ by

$$
\rho=\rho_{c} w^{n_{r}}
$$

where $\rho_{c}$ denotes the energy density at the center (from now on the subscript $c$ indicates that the variable is evaluated at the center), we may rewrite (24) as

$$
P_{r}=K \rho_{c}^{\gamma_{r}} w^{n_{r} \gamma_{r}}=P_{r c} w^{1+n_{r}},
$$

with $P_{r c}=K \rho_{c}^{\gamma_{r}}$. Note that from $(26)$, we can write

$$
P_{r}^{\prime}=P_{r c}\left(1+n_{r}\right) w^{n_{r}} w^{\prime},
$$

so that 18 can be written as

$$
2 P_{r c}\left(1+n_{r}\right) w^{\prime}+\left(P_{r c} w+\rho_{c}\right) \nu^{\prime}-4 \frac{\Delta}{r w^{n_{r}}}=0 .
$$

Next, dividing by $\rho_{c}$ and defining $q_{c}=\frac{P_{r c}}{\rho_{c}}$, we obtain

$$
2 q_{c}\left(1+n_{r}\right) w^{\prime}+\left(q_{c} w+1\right) \nu^{\prime}-4 \frac{\Delta}{r \rho_{c} w^{n_{r}}}=0,
$$

from where

$$
\nu^{\prime}=\frac{4 \Delta}{r \rho_{c} w^{n_{r}}\left(q_{c} w+1\right)}-\frac{2 q_{c}\left(1+n_{r}\right)}{q_{c} w+1} w^{\prime} .
$$

The integration of 30 produces

$$
\nu=\nu_{c}+\frac{4}{\rho_{c}} \int_{0}^{r} \frac{\Delta d r}{r\left(q_{c} w+1\right) w^{n_{r}}}-2\left(1+n_{r}\right) \log \left(\frac{q_{c} w+1}{q_{c}+1}\right) .
$$

To obtain $\nu_{c}$ we can use the boundary condition 14, from where

$$
\nu_{c}=\log \left(\frac{1-\frac{2 M}{r_{\Sigma}}}{\left(1+q_{c}\right)^{2\left(1+n_{r}\right)}}\right)-\frac{4}{\rho_{c}} \int_{0}^{r_{\Sigma}} \frac{\Delta d r}{r\left(q_{c} w+1\right) w^{n_{r}}} .
$$

Replacing 32 in 31 we obtain

$$
\nu=\log \left(\frac{1-\frac{2 M}{r_{\Sigma}}}{\left(1+q_{c} w\right)^{2\left(1+n_{r}\right)}}\right)-\frac{4}{\rho_{c}} \int_{r}^{r_{\Sigma}} \frac{\Delta d r}{r\left(q_{c} w+1\right) w^{n_{r}}},
$$

whereas replacing 20 and $(30)$ in $(11)$, produces

$$
\begin{aligned}
& q_{c} w \frac{d m}{d r}+\frac{m}{r}+q_{c}\left(1+n_{r}\right) \frac{r}{1+q_{c} w} \frac{d w}{d r}\left(1-\frac{2 m}{r}\right) \\
& -\frac{2 \Delta}{\rho_{c}} \frac{\left(1-\frac{2 m}{r}\right)}{\left(1+q_{c} w\right) w^{n_{r}}}=0 .
\end{aligned}
$$

Let us now introduce the following dimensionless variables

$$
\begin{aligned}
\eta & =\frac{m}{4 \pi \rho_{c} \alpha^{3}}, \\
r & =\alpha x \\
\alpha^{2} & =\epsilon \frac{\left(n_{r}+1\right) q_{c}}{4 \pi \rho_{c}},
\end{aligned}
$$

in terms of which 34 can be written as

$$
\begin{aligned}
& {\left[\frac{\epsilon x-2 q_{c}\left(1+n_{r}\right) \eta}{1+q_{c} w}\right]\left[x w^{\prime}-\frac{2 \Delta}{\rho_{c} q_{c}\left(1+n_{r}\right) w^{n_{r}}}\right]} \\
& +\eta+q_{c} x w \eta^{\prime}=0,
\end{aligned}
$$

where $\eta^{\prime}=x^{2} w^{n_{r}}$ and either $\epsilon=+1$ for $n_{r}>-1$ or $\epsilon=-1$ for $n_{r}<-1$. Please notice that from now on the prime denotes derivative with respect to the variable $x$.

It is worth noticing that after restoring the speed of light, we have

$$
q_{c}=\frac{P_{c}}{\rho_{c} c^{2}}
$$

implying that in the Newtonian limit (i.e. $c \rightarrow \infty$ ), we have $q_{c} \rightarrow 0$, producing

$$
x\left[x w^{\prime}-\frac{2 \Delta}{P_{r c}\left(1+n_{r}\right) w^{n_{r}}}\right]+\eta=0 .
$$


Then, deriving 40 and using $\eta^{\prime}=x^{2} w^{n_{r}}$ we obtain

$$
\begin{aligned}
& w^{\prime \prime}+\frac{2}{x} w^{\prime}+w^{n_{r}} \\
& -\frac{2}{\left(1+n_{r}\right) P_{r c} w^{n_{r}} x}\left[\Delta^{\prime}+\frac{\Delta}{x}-n_{r} \frac{w^{\prime}}{w} \Delta\right]=0,
\end{aligned}
$$

which is the corresponding equation obtained in the Newtonian case 1 .

Let us now consider the case $n= \pm \infty(\gamma=1)$ which leads to

$$
P_{r}=K \rho \text {. }
$$

Defining the dimensionless variable $w$ by

$$
\rho=\rho_{c} e^{-w},
$$

(42) reads

$$
P_{r}=K_{r} \rho_{c} e^{-w}=P_{r c} e^{-w} .
$$

Then, replacing in 18 we obtain

$$
\frac{d \nu}{d r}=\frac{2 P_{r c}}{\rho_{c}+P_{r c}} \frac{d w}{d r}+\frac{4}{\left(\rho_{c}+P_{r c}\right) r} \Delta e^{w},
$$

or using 20, 111) and $q_{c}=P_{r c} / \rho_{c}$

$$
\begin{gathered}
\frac{2 e^{w} \Delta}{\rho_{c}\left(1+q_{c}\right)}\left(1-\frac{2 m}{r}\right)+\frac{q_{c} r}{1+q_{c}}\left(1-\frac{2 m}{r}\right) \frac{d w}{d r} \\
-\frac{m}{r}-q_{c} \frac{d m}{d r}=0 .
\end{gathered}
$$

Finally, in terms of the dimensionless quantities defined in (35), 36 and 37) the Lane-Emden equation reads

$$
\begin{gathered}
{\left[\frac{2}{q_{c} \rho_{c}} e^{w} \Delta+x w^{\prime}\right]\left[x-\frac{q_{c}}{1+q_{c}} \eta\right]} \\
-\eta-q_{c} x \eta^{\prime}=0,
\end{gathered}
$$

with $\eta^{\prime}=x^{2} e^{-w}$. It is worth noticing that in the limit $q_{c} \rightarrow 0$ the solution reduces to

$$
w^{\prime \prime}+\frac{2}{x} w^{\prime}+\frac{2}{P_{r c} x} e^{w}\left(\Delta^{\prime}+\frac{\Delta}{x}+\Delta w^{\prime}\right)=e^{-w},
$$

which coincides with the Newtonian limit reported in [1].

\section{THE DOUBLE POLYTROPE}

As previously commented, in order to solve the problem of the general relativistic polytrope for anisotropic matter, additional information (besides (3) must be provided. In this work this information is supplied by the assumption that tangential pressure also satisfies a polytropic equation of state. Again, due to the bifurcation appearing at $\gamma=1$, we shall consider separately the cases $\gamma \neq 1$ and $\gamma=1$ mentioned in the previous section. However, since we have now two polytropic equations of state we shall clearly differentiate two polytropic exponents (indexes) $\gamma_{r}, \gamma_{\perp}\left(n_{r}, n_{\perp}\right)$, one for each polytrope. Thus, three possible cases may be considered.

\section{A. Case 1: Both polytropes with $\gamma \neq 1$}

In this subsection we shall assume that $\gamma_{r} \neq 1, \gamma_{\perp} \neq 1$, and the tangential pressure satisfies the polytropic equation of state

$$
P_{\perp}=K_{\perp} \rho^{\gamma_{\perp}}
$$

whereas the radial pressure satisfies 3 .

Fom the above

$$
\Delta=K_{\perp} \rho^{\gamma_{\perp}}-K_{r} \rho^{\gamma_{r}} .
$$

Introducing $w$ by

$$
\rho=\rho_{c} w^{n_{r}},
$$

and replacing (51) in 50 we may write

$$
\Delta=P_{r 0}\left(w^{n_{r} \gamma_{\perp}}-w^{n_{r} \gamma_{r}}\right)=\rho_{c} q_{c} w^{n_{r}}\left(w^{\theta}-w\right),
$$

where $\theta=n_{r} / n_{\perp}$. Within this model the Lane-Emden equation reads

$$
\begin{aligned}
& {\left[\frac{x-2 q_{c}\left(1+n_{r}\right) \eta}{1+q_{c} w}\right]\left[x w^{\prime}-\frac{2\left(w^{\theta}-w\right)}{1+n_{r}}\right]} \\
& +\eta+q_{c} x w \eta^{\prime}=0,
\end{aligned}
$$

with $\eta^{\prime}=x^{2} w^{n_{r}}$.

In figure 1 it is shown the integration of Eq. (53) for the values of the parameters indicated in the figure legend. Note that $w$ is monotonously decreasing as expected, and

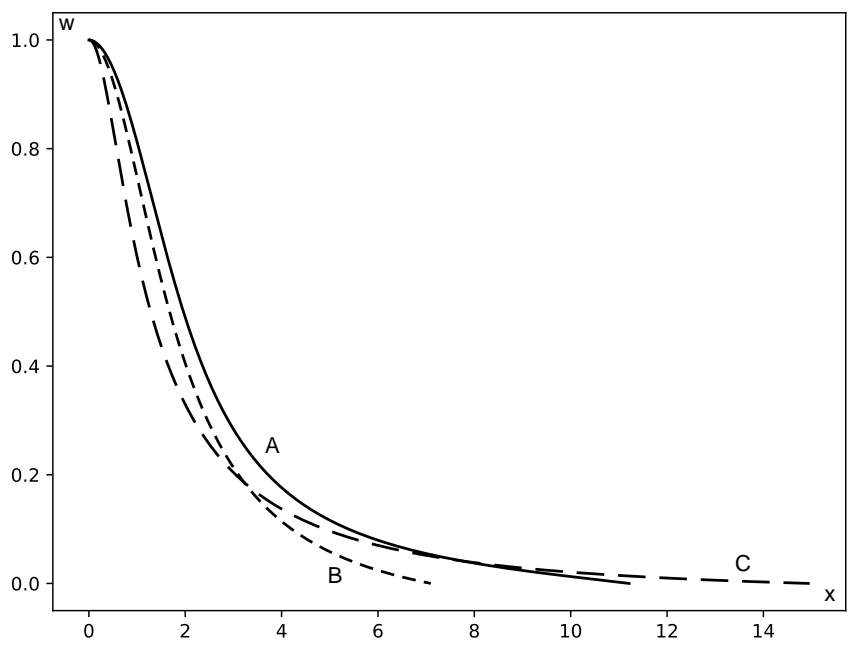

FIG. 1: Case 1. $w$ as a function of $x$ for $n_{r}=3, q_{c}=0.1$ and $\theta=0.5$ (short dashed line), $\theta=2$ (long dashed line) and $\theta=4$ (solid line)

the radial pressure $P_{r}$ vanishes at the surface as required by the continuity of the second fundamental form.

It will be useful to calculate the Tolman mass, which is a measure of the active gravitational mass [28, defined by

$$
m_{T}=\frac{1}{2} r^{2} e^{\frac{\nu-\lambda}{2}} \nu^{\prime}
$$




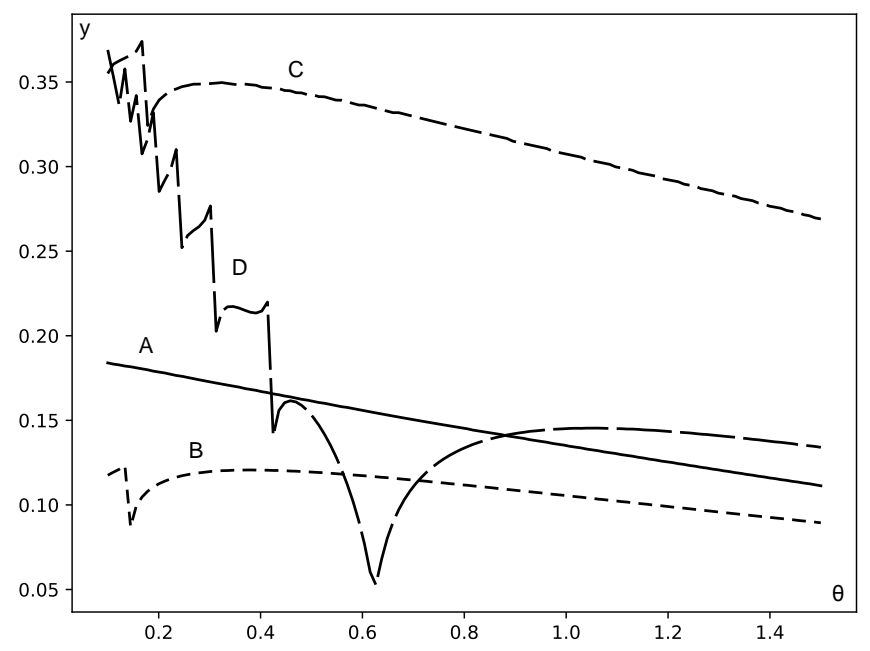

FIG. 2: Case 1. Surface potential $y$ as a function of the anisotropy parameter $\theta$ for pairs $\left(q_{c}, n_{r}\right)$. A: $(0.1,1.0)$ (solid line), B: $(0.1,2.0)$ (short-dashed line), C: $(1.0,1.0)$ (mediumdashed line), D: (1.0,2.0) (long-dashed line).

Alternatively we can calculate the Tolman mass from the equivalent expression [5],

$$
m_{T}=e^{\frac{\nu+\lambda}{2}}\left(m+4 \pi r^{3} P_{r}\right) .
$$

Now, in order to obtain $\nu$ we proceed as follows. First, the TOV equation (18) can be written as

$$
P_{r c}\left(1+n_{r}\right) d w=-\frac{1}{2} \rho_{c}\left(1+q_{c} w\right) d \nu+\frac{2 P_{r c}}{r}\left(w^{\theta}-w\right) d r
$$

from where,

$$
\begin{aligned}
& \int_{w(r)}^{w\left(r_{\Sigma}\right)} \frac{d w}{\left(1+q_{c} w\right)}=-\frac{\rho_{c}}{2 P_{r c}} \frac{1}{\left(1+n_{r}\right)} \int_{\nu(r)}^{\nu\left(r_{\Sigma}\right)} d \nu \\
& +\frac{2}{1+n_{r}} \int_{r}^{r_{\Sigma}} \frac{w^{\theta}-w}{\left(1+q_{c} w\right) r} d r .
\end{aligned}
$$

Next, defining $G(r)$ as

$$
G(r) \equiv \int_{r}^{r_{\Sigma}} \frac{w^{\theta}-w}{\left(1+q_{c} w\right) r} d r
$$

the integration of Eq. 57) produces

$$
-2\left(1+n_{r}\right) \log \left(1+q_{c} w\right)=\nu(r)-\nu\left(r_{\Sigma}\right)+4 q_{c} G(r)
$$

from where we obtain

$$
e^{\nu}=\frac{1-\frac{2 M}{r_{\Sigma}}}{\left(1+q_{c} w\right)^{2\left(1+n_{r}\right)} e^{4 q_{c} G(r)}} .
$$

Finally using (20) (35), 36), 37) and (60) in (55) we arrive at

$$
\eta_{T}=\left(\frac{1-2 y}{1-2 \epsilon q_{c}\left(1+n_{r}\right) \frac{\eta}{x_{\Sigma} z}}\right)^{1 / 2} \frac{\eta+q_{c} x_{\Sigma}^{3} z^{3} w^{1+n_{r}}}{\left(1+q_{c} w\right)^{1+n_{r}} e^{2 q_{c} G(z)}},
$$

where

$$
\begin{aligned}
\eta_{T} & =\frac{m_{T}}{4 \pi \rho_{c} \alpha^{3}} \\
y & =\frac{M}{r_{\Sigma}}, \\
z & =\frac{x}{x_{\Sigma}},
\end{aligned}
$$

and

$$
G(z)=\int_{z}^{1} \frac{w^{\theta}-w}{\left(1+q_{c} w\right) z^{\prime}} d z^{\prime}
$$

The parameter $y$ ("the surface potential") which measures the degree of compactness is plotted in figure 2 as function of the anisotropy parameter $\theta$ for different duplets $\left(q_{c}, n_{r}\right)$.

Figure 3 displays the Tolman mass (normalized by the total mass), for the case 1 , as function of $z$ for the selection of values of the parameters indicated in the legend. The behaviour of the curves is qualitatively the same for a wide range of values of the parameters. This figure deserves a detailed analysis.

Indeed, observe that as we move from the less compact configuration (curve D) to the more compact one (curve A), the Tolman mass tends to concentrate on the outer regions of the sphere, except for curves $\mathrm{B}, \mathrm{C}, \mathrm{D}$ at the innermost regions (see some comments on this point in the last section). In its turn, more compact configurations correspond to smaller values of the parameter that measures the anisotropy and smaller values of the Tolman mass in the inner regions. In other words, for this case, smaller values of $\theta$ (corresponding to more compact configurations) reach stability by reducing the active gravitational mass in the inner regions. Therefore, it may be inferred from this figure that more stable configurations correspond to smaller values of $\theta$ since they are associated to a sharper reduction of the Tolman mass in the inner regions.

\section{B. Case 2: $\gamma_{r}=1$ and $\gamma_{\perp} \neq 1$}

In this case, besides considering $P_{r}=K_{r} \rho$ with $\rho=$ $\rho_{c} e^{-w}$ we assume

$$
P_{\perp}=K_{\perp} \rho^{1+\frac{1}{n_{\perp}}},
$$

from where the anisotropy factor reads

$$
\Delta=P_{r c} e^{-w}\left(e^{-\frac{w}{n_{\perp}}}-1\right) .
$$




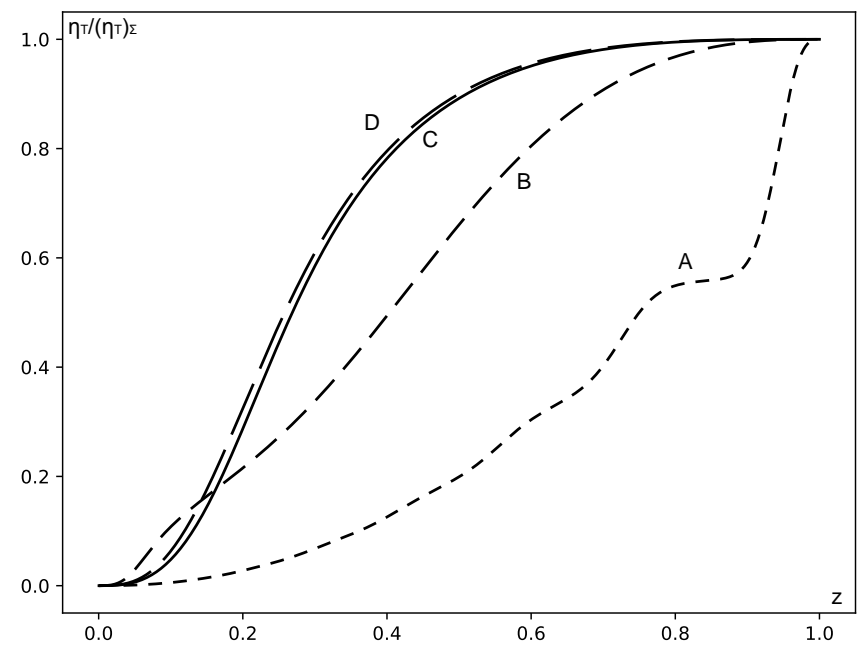

FIG. 3: Case 1. $\eta_{T} /\left(\eta_{T}\right)_{\Sigma}$ as a function of $z$ for $n_{r}=2.0, q_{c}=$ 1.0 and different values of $y(\theta)$. A: 0.3690(0.1) (short-dashed line), B: 0.1550(0.5) (medium-dashed line), C: 0.1449(1.0) (solid line-isotropic case), D: 0.1341(1.5) (long-dashed line). Values of $y$ are read from Fig. 2 .

From the above, the Lane-Emden equation (47) becomes

$$
\begin{gathered}
{\left[x w^{\prime}+2\left(e^{-\frac{w}{n_{\perp}}}-1\right)\right]\left(x-\frac{q_{c}}{1+q_{c}} \eta\right)} \\
-\eta-q_{c} x \eta^{\prime}=0
\end{gathered}
$$

with $\eta^{\prime}=x^{2} e^{-w}$.

In figure 4 it is shown the integration of Eq. 68 for the values of the parameters indicated in the figure legend.

As it is apparent from this figure, these configurations are unbounded and therefore it is meaningless to define a surface potential or the total mass.

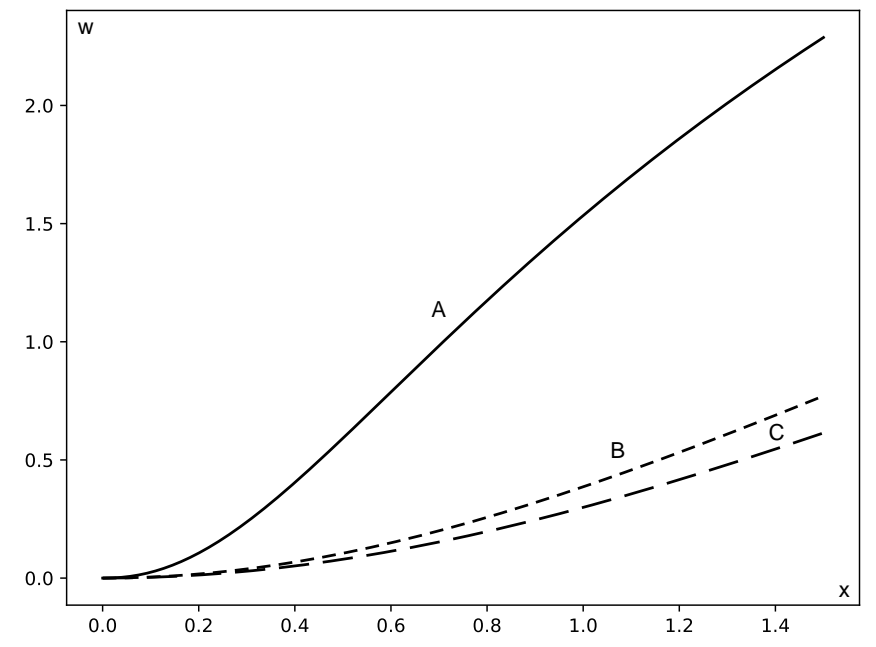

FIG. 4: Case 2. $w$ as a function of $x$ for $q_{c}=0.1$, and $n_{\perp}=1$ (short dashed line), $n_{\perp}=2$ (long dashed line) and $n_{\perp}=3$ (solid line)

\section{Case 3: $\gamma_{r} \neq 1 \gamma_{\perp}=1$}

In this case we assume $P_{r}=K_{r} \rho^{1+\frac{1}{n_{r}}}$ with $\rho=\rho_{c} w^{n_{r}}$ and

$$
P_{\perp}=K_{\perp} \rho
$$

from where the anisotropy reads

$$
\Delta=P_{r c} w^{n_{r}}(1-w) .
$$

Then, 38 becomes

$$
\begin{gathered}
{\left[\frac{\epsilon x-2 q_{c}\left(1+n_{r}\right) \eta}{1+q_{c} w}\right]\left[x w^{\prime}-\frac{2(1-w)}{\left(1+n_{r}\right)}\right]} \\
+\eta+q_{c} x w \eta^{\prime}=0,
\end{gathered}
$$

with $\eta^{\prime}=x^{2} w^{n_{r}}$. In figure 5 it is shown the integration of Eq. (71) for the values of the parameters indicated in the figure legend.

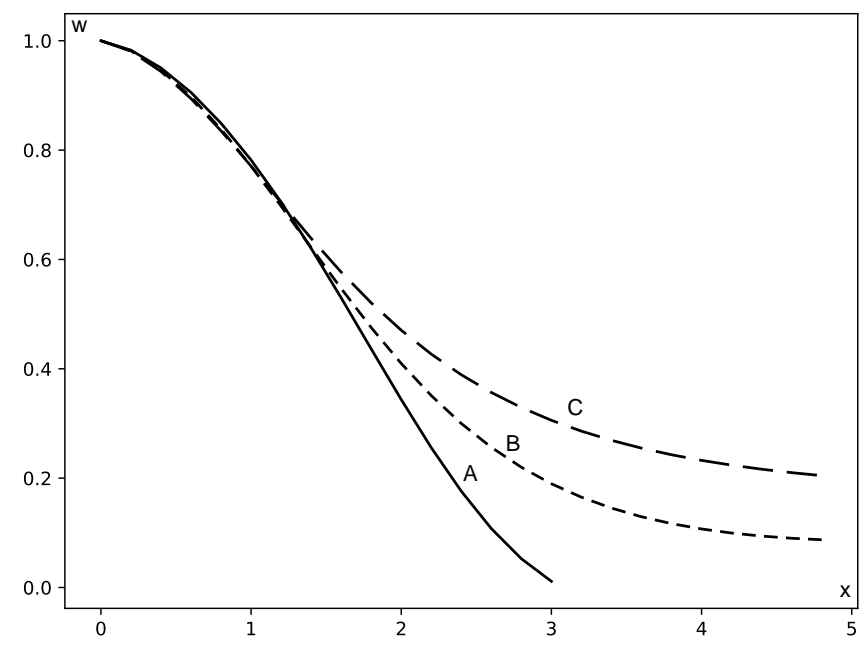

FIG. 5: Case 3. $w$ as a function of $x$ for $q_{c}=0.1$, and $n_{r}=1$ (short dotted line), $n_{r}=2$ (long dotted line) and $n_{r}=3$ (solid line)

Next, the TOV equation may be rewritten for this case as,

$$
P_{r c}\left(1+n_{r}\right) d w=-\frac{1}{2} \rho_{c}\left(1+q_{c} w\right) d \nu+\frac{2 P_{r c}}{r}(1-w) d r,
$$

and integrating we obtain

$$
\begin{aligned}
& \int_{w(r)}^{w\left(r_{\Sigma}\right)} \frac{d w}{\left(1+q_{c} w\right)}=-\frac{\rho_{c}}{2 P_{r c}} \frac{1}{\left(1+n_{r}\right)} \int_{\nu(r)}^{\nu\left(r_{\Sigma}\right)} d \nu \\
& +\frac{2}{1+n_{r}} \int_{r}^{r_{\Sigma}} \frac{1-w}{\left(1+q_{c} w\right) r} d r .
\end{aligned}
$$

The above equation is the equivalent to (57), for this case. They differ in the last term corresponding to the 


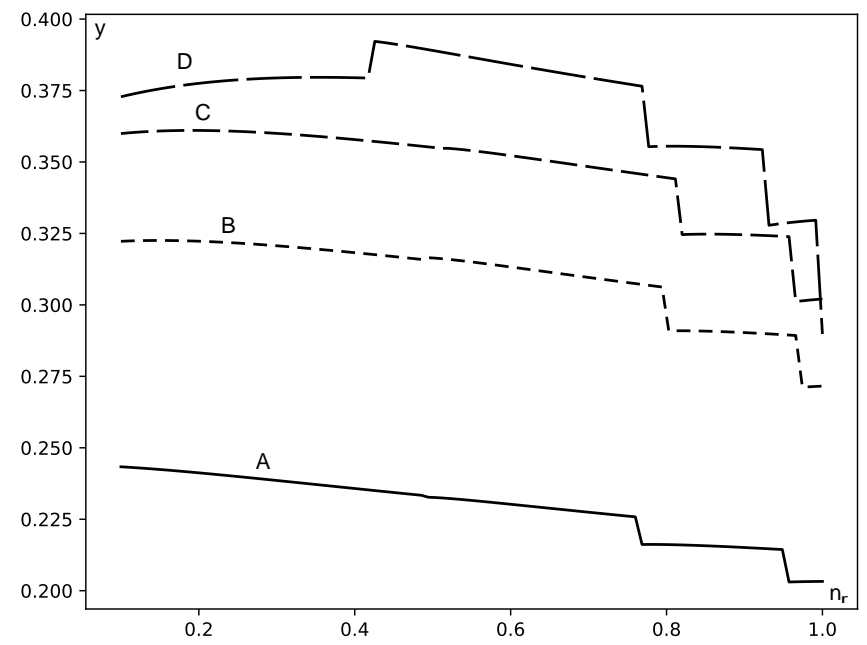

FIG. 6: Case 3. Surface potential $y$ as a function of the polytropic index $n_{r}$ for different $q_{c}$ values. A: $q_{c}=0.10$ (solid line), B: $q_{c}=0.18$ (short-dashed line), C: $q_{c}=0.24$ (mediumdashed line), D: $q_{c}=0.30$ (long-dashed line).

function $G(r)$. Thus, redefining the function $G(r)$ for the case 3 by

$$
G(r) \equiv \int_{r}^{r_{\Sigma}} \frac{1-w}{\left(1+q_{c} w\right) r} d r
$$

and retracing the same steps as in the case 1 , we found for the Tolman mass in this case the same expression (61), but with a different function $G(z)$, which now reads

$$
G(z)=\int_{z}^{1} \frac{1-w}{\left(1+q_{c} w\right) z^{\prime}} d z^{\prime} .
$$

The surface potential $y$ and the normalized Tolman mass, for a selection of values of the parameters are plotted in figures 6 and 7 respectively. The exhibited behavior of these variables are qualitatively the same as for a wide range of values of the parameters. Also, the conclusions extracted from figure 7 are basically the same as the ones reached at from the analysis of the case 1.

\section{CONCLUSIONS}

Motivated by the relevance of pressure anisotropy in the structure of self-gravitating objects, and by the fact that polytropes represent fluid systems with a wide range of applications in astrophysics (e.g. Fermi fluids), we have described hereby a general framework for the modeling of general relativistic polytropes in the presence of anisotropic pressure, when both pressures satisfy a polytropic equation of state. Thus, this work may be interpreted as a natural generalization of the approach described in [1, to the relativistic regime. As mentioned

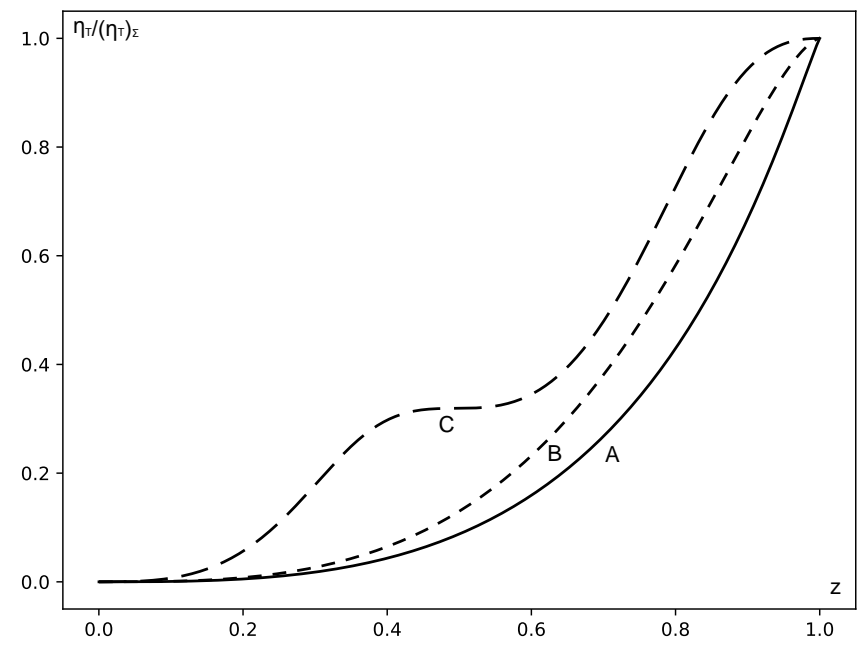

FIG. 7: Case 3. $\eta_{T} /\left(\eta_{T}\right)_{\Sigma}$ as a function of $z$ for $q_{c}=0.3$ and different values of $y\left(n_{r}\right)$. A: $0.3728(0.1)$ (solid line), B: 0.3889(0.5) (short-dashed line), C: 0.2898(1.0) (mediumdashed line). Values of $y$ are read from Fig. 6

in the Introduction, such an extension is mandatory if one has to deal with ultra compact objects such as neutron stars, where general relativistic effects cannot be neglected.

Since the inclusion of pressure anisotropy implies an additional degree of freedom, the integration of the ensuing Lane-Emden equation, in the general case, requires additional information. We have supplied such additional information by assuming that both pressures satisfy a polytropic equation of state. The motivation to adopt such an assumption is provided by the simple fact that for small anisotropies it is always a good approximation. For large anisotropies it is just an heuristic assumption whose validity will be confirmed (or denied) from its application to specific problems.

It should be recalled that for each polytrope there are two possible polytropic equations of state leading to the same Newtonian limit, depending on whether we use the energy density or the baryonic mass density. Therefore for two polytropes, as is the case in this work, we have in principle four possible cases. We have discussed only the case represented by (3) and (4) (i.e. we use the energydensity for both polytopes). The treatment of the remaining three cases is very similar and, therefore, for simplicity we have omitted here their description.

Depending on whether $\gamma=1$ or $\gamma \neq 1$ we could differentiate three possible cases. It should be noticed that there are only three possible cases since the case $\gamma_{r}=\gamma_{\perp}$ leads to the isotropic pressure case $P_{r}=P_{\perp}$. We have integrated the Lane-Emden equations for these three cases, for a very large set of values of the parameters. However, only a very specific set for each case is exhibited, since the qualitative behaviour of the system does not change much for a wide range of values of the parameters.

Although the main reason to present such models was 
not to describe any specific astrophysical scenario, but to illustrate the applications of our approach, the obtained models exhibit some interesting features which deserve to be commented.

Thus we observe in figure 1 that bounded configurations exist for a range of values of the parameters, out of which the configurations are unbounded. However due to the existence of a larger number of parameters than in the isotropic case, the conditions for the existence of finite radius distributions are more involved than in the latter case. The same happens for the case 3, depicted in figure 5. In the case 2, instead, all configurations are unbounded, as illustrated by figure 4 . This is an expected result since this case corresponds to an isothermal gas.

Figures 3 and 7 illustrate the "strategy" adopted by the fluid distribution to keep the equilibrium; it tries to concentrate the Tolman mass in the outer regions. This behaviour was already observed for different families of anisotropic politropes discussed in [16. Two remarks are in order at this point:

- The "anomalous" behavior shown for the innermost regions in figure 3 is related to the extreme (maximal) value of $q_{c}$ that has been used for this fig- ure $\left(q_{c}=1\right)$, it corresponds to the stiff equation of state $P_{r}=\rho$, which is believed to describe ultradense matter 29]. For smaller values of $q_{c}$ the above mentioned behaviour does not appear.

- The efficiency to diminish the Tolman mass in the inner regions and to concentrate it in the outer ones, depends on the anisotropic factor, which brings out the role played by the anisotropy in the stability of the fluid configuration [30].

Finally we would like to call the attention to the potential application of the approach presented here to the study of super-Chandrasekhar white dwarfs which may attain masses of the order of $2.8 M_{\odot}$, and are modeled resorting to a polytropic equation of state (see 31 and references therein). For such configurations it is evident that general relativistic effects as well as the inclusion of pressure anisotropy, are unavoidable. Nevertheless, care must be exercised with the fact that some of the physical phenomena present in such configurations (e.g. very strong magnetic fields) could break the spherical symmetry, implying thereby that our approach should be taken, in this case, as an approximation.
[1] G. Abellán, E. Fuenmayor and L. Herrera, Phys. Dark Univ. 28, 100549 (2020).

[2] L. Herera and W. Barreto, Phys. Rev D 87, 087303 (2013).

[3] S. Chandrasekhar, An Introduction to the Study of Stellar Structure (University of Chicago, Chicago, 1939).

[4] R. Kippenhahn and A. Weigert, Stellar Structure and Evolution (Springer Verlag, Berlin, 1990).

[5] L. Herrera and N.O. Santos, Phys. Rep. 286, 53 (1997).

[6] L. Herrera, Phys. Rev. D 101, 104024 (2020).

[7] R. Tooper, Astrophys. J., 140, 434 (1964).

[8] R. Tooper, Astrophys. J., 142, 1541 (1965).

[9] R. Tooper, Astrophys. J., 143, 465 (1966).

[10] S. Bludman, Astrophys. J., 183, 637 (1973).

[11] U. Nilsson and C. Uggla, Ann. Phys., 286, 292 (2000).

[12] H. Maeda, T. Harada, H. Iguchi and N. Okuyama, Phys. Rev. D, 66, 027501 (2002).

[13] L. Herrera and W. Barreto, Gen. Relativ. Gravit., 36, 127 (2004).

[14] X. Y. Lai and R. X. Xu, Astropart. Phys., 31, 128 (2009).

[15] S. Thirukkanesh and F. C. Ragel, Pramana J. Phys., 78, 687 (2012).

[16] L. Herrera and W. Barreto, Phys. Rev. D 88, 084022 (2013).

[17] L. Herrera, A. Di Prisco, W. Barreto and J. Ospino, Gen. Relativ. Gravit., 46, 1827 (2014).
[18] S. A. Ngubelanga and S. D. Maharaj, Eur. Phys. J. P.,130, 211, (2015).

[19] T. Harko and M. K. Mak, Astrophys. Space Sci.,361, 283 (2016).

[20] L. Herrera, E. Fuenmayor, and P. Leon, Phys. Rev. D 93, 024047 (2016).

[21] H.-C. Kim, Phys. Rev., D 96, 064053 (2017).

[22] S. A. Mardan and M. Azam, Eur. Phys. J. C, 77, 385 (2017).

[23] M. Moussa, Ann. Phys.,385, 347 (2017).

[24] M. Z. Bhatti and Z. Tariq, Eur. Phys. J. P. 134, 521 ( 2019).

[25] R. Roy, Pramana, 92, 63, (2019).

[26] S. A. Mardan, A. A. Siddiqui, I. Noureen, and R. N. Jamil, Eur. Phys. J. P., 135 , 3 (2020).

[27] M. Z. Bhatti and Z. Tariq, Phys. Dark Univ., 28,100482, (2020).

[28] L. Herrera, W. Barreto, A. Di. Prisco and N. O. Santos, Phys. Rev. D 65, 104004 (2002).

[29] Ya. B. Zeldovich, Zh. Eksp. Teor. Fiz. 41, 1609 (1969) [Sov. Phys. JETP 14, 1143 (1962)]

[30] R. Chan, L. Herrera and N. O. Santos, Month. Not. Roy. Astron. Soc. 265, 533 (1993).

[31] S. Kalita, B. Mukhopadhyay, T. Mondal, and T. Bulik, arXiv:2004.13750v1[astro-ph.HE] 